far in my career, I have not chosen to share my lack of religious belief with any of my patients. This patient had recently had delusions with religious content. Knowing that I do not have a religion might have all sorts of implications for our therapeutic relationship. Also, the patient may tell the other patients on my ward, and the staff, with further potential implications for therapeutic and professional relationships.

Colleagues giving evidence in a civil or criminal court in the presence of one of their patients may wish to avoid such a disclosure. Although there is no way of giving oral evidence without making an affirmation or taking an oath (without risking contempt of court), I think if I was in a similar situation again I would let the clerk know in advance that I would like to make the affirmation. The clerk would then have no need to ask me about my religion and thus draw attention to my atheism in court.

This incident also made me wonder whether it is right that witnesses have to reveal their religion in open court before they can give evidence. Witnesses often do not have a choice whether to give evidence. 'Religious beliefs or other beliefs of a similar nature' are considered sensitive personal data in the Data Protection Act 1998 because their handling requires particular care, and cannot be 'processed' without special conditions being met. I cannot find any evidence whether rates of lying in court differ when the affirmation or the oath is used; in my opinion it is unlikely to make any difference. Perhaps the current oath system involves an unnecessary and unfair forced disclosure on the part of witnesses.

Catherine Penny, Locum Consultant Forensic Psychiatrist, Oxleas NHS Foundation Trust, UK, email: cpenny@nhs.net

doi: $10.1192 / \mathrm{pb} .36 .4 .156 a$

\section{Five simple questions to predict violence in psychiatric patients}

Forensic psychiatry is principally concerned with assessing and managing the risk to others (usually of violence) by people with a mental disorder. A variety of lengthy risk assessment instruments help consolidate this expertise, but these instruments do not find favour in day-to-day psychiatry.

Based on the work of Fazel and colleagues, ${ }^{1,2}$ we set out to determine whether five 'yes or no' questions (male gender; less than 32 years old; previous criminal convictions; and comorbid alcohol misuse and drug misuse) could predict later actual physical violence to others. We analysed case notes on consecutively discharged patients from a medium secure forensic unit (52 patients, 46 male); an out-patient addictions service (51 patients; 26 male); and a crisis resolution and home treatment service ( 25 patients, 17 male), in a 'pseudoprospective' method for a record of physical violence after applying these five questions as a screen to the case records 5 years earlier, from January 2006. Records with insufficient detail or length of history were excluded, and the screen was viewed as a positive predictor if three or more questions were answered 'yes'.

We found 30 (of 128) patients were violent in the 5 years studied, with $83 \%$ being predicted as violent by our screen (sensitivity), and a false negative rate of $17 \%$. The positive predictive value was poor at $38 \%$ but the negative predictive value (i.e. that a negative prediction was correct) was impressive at $92 \%$. The factors predicting later violence were being male (93\%); having a history of violence $(80 \%$, not a 'Fazel' question); a history of drug misuse (77\%); a prior criminal conviction (70\%); a history of alcohol misuse (60\%); poor treatment adherence (52\%, not a 'Fazel' question); and being less than 32 years old (50\%). A history of self-harm was only seen in $20 \%$ of those who were violent later.

The rates of 5 -year violence in the three separate groups were $35 \%$ in the forensic sample, $6 \%$ in addictions, and $36 \%$ in the acute community crisis resolution home treatment group. We acknowledge the preponderance of males in our sample will skew the results, given it is a screening question.

Our results raise two interesting points. First, that these five simple questions might aid clinical decision-making concerning which patients will not pose a risk of later violence, but does not elucidate prediction on who will become violent. This screen might therefore be useful as part of a stepped approach in a busy clinical environment when considering who to refer for more in-depth assessment. Second, as Turner \& Salter have already noted, ${ }^{3}$ we conclude it is hard to define who is 'a forensic patient' when we compare patterns across our three samples.

1 Fazel S, Långström N, Hjern A, Grann M, Lichtenstein P. Schizophrenia, substance abuse, and violent crime. JAMA 2009; 301: 2016-23.

2 Singh JP, Fazel S. 'Developing a violence screening instrument for patients with schizophrenia'. Paper presented at the XXXII International Congress on Law and Mental Health, Berlin, Germany, July 2011.

3 Turner T, Salter M. Forensic psychiatry and general psychiatry: re-examining the relationship. Psychiatrist 2008; 32: 2-6.

Mark Taylor is Consultant Psychiatrist, Ballenden House, NHS Lothian, Edinburgh, email: marktaylor2@nhs.net; Adam Groves, Fiona McCusker, Natalie Lee, Johanna Bradley, Gianluca Alonzi, Claire Murphy, Rebecca Miles, Samantha Ellis and Ellie Richardson are all medical students at the University of Edinburgh, UK.

doi: $10.1192 / p b .36 .4 .157$ 